Proceedings

\title{
New High-throughput reactor for biomass valorisation
}

\author{
Irene Malpartida ${ }^{1}$, Pedro Maireles-Torres ${ }^{2}$, Valentin Lair ${ }^{1}$, Samy Halloumi ${ }^{1}$, Julien Thiel ${ }^{1}$, François Lacoste ${ }^{1}$ \\ 1 Deasyl S.A., Plan Les Ouates, Geneva, Switzerland; i.malpartida@deasyl.ch \\ 2 Universidad de Málaga, Departamento de Química Inorgánica, Cristalografía y Mineralogía, 29071 Málaga, Spain; \\ maireles@uma.es \\ * Correspondence: i.malpartida@deasyl.ch. \\ + Presented at the 6th International Electronic Conference on Sensors and Applications, \\ 15-30 November 2019; Available online: https://ecsa-6.sciforum.net/
}

\begin{abstract}
The development of an innovative and sustainable high-throughput reaction platform allows to optimize a wide range of chemical processes (materials synthesis, catalysis, among others) to tackle the Green Deal. This tool unifies for the first time the benefits of mechanical energy, thermal and pressure activation, continuous flow with an induction in-situ heating system, facilitating the incorporation of inputs (liquids, solids and gases) with controlled pressure. As result of synergistic effect of this simultaneous activation, this technology will: i) shorten reaction times; ii) decrease temperature; iii) improve reactions kinetics as mass transfer limitations are reduced; iv) minimize the use of solvents; v) decrease the reaction steps; v) increase volume treated, enabling a real scale-up; and vi) enhance the yields and/or selectivity. This new high-throughput reactor is used for the synthesis of Calcium diglyceroxide (CaDG), minimizing the reaction steps and cost, to obtain a pure CaDG. This heterogeneous catalyst is used for biodiesel production and valorisation of the glycerol generated as by-product. An efficient synthesis protocol of CaDG has been developed, requiring shorter time, without heating and no need of solvent. This new process facilitates the oil-methanol mixing in the transesterification process, thus minimizing the mass transfer limitations associated to the immiscibility of reactants. In addition, this process has been optimised by using CaDG as solid catalyst.
\end{abstract}

Keywords: biomass; biodiesel; biofuel; calcium diglyceroxide; heterogeneous catalysis; glycerol valorisation;

\section{Introduction}

Depletion of fossil fuels constitutes nowadays one of the most critical problematics in all the scientific agendas of the world, including the European Green Deal and Sustainable Development Goals of the United Nations. Looking forward to avoid a crisis of chemical and fuels supplies, without affecting current living standards, biorefinery has emerged as an outstanding alternative to replace the petro-based industry. The use of biomass residues, as feedstock for biorefinery, represents an inexpensive option also promoting environmental sustainability and helping with the transition to climate neutrality. Biomass valorisation is also an opportunity with economic benefits, especially for companies generating biomass wastes, boosting the integration of circular economy and green chemistry principles [1].

However, traditional methods for their synthesis are neither atom-efficient nor sustainable [2]. Therefore, the development of greener pathways are highly necessary. The use of renewable biomass feedstock for, sustainable processes must also consider the use of catalysis, the reduction of solvents and additional reagents, the decrease of reaction times, minimization of energy requirements and scalable processes.

In this context, mechanochemistry represents an environmentally friendly strategy, which still requires a lot of efforts for its establishment as a consolidated approach for organic synthesis. After decades of study, mechanochemistry has demonstrated to: i) simplify work-up protocols, ii) allow safer processes and iii) reduce waste improving energy and cost-efficiencies. Mechanochemical methods could certainly contribute to re-thinking organic chemistry from a greener perspective and translate it in a more sustainable chemical industry [3]. 
The development of an innovative high-throughput reaction platform that will allow to uniquely optimize a wide range of chemical processes to contribute to tackle the European Green Deal [4]. This tool will unify for the first time the benefits from mechanical energy, thermal and pressure activation in continuous flow. In addition to taking advantage of the powerful synergy obtained from this innovative combination, a tailored in-situ heating system will allow the incorporation of inputs (liquids, solids and gas) with controlled pressure. This innovative reaction platform will allow to efficiently carry out a large variety of processes, providing a ground-breaking sustainable, versatile and scalable alternative to currently available technologies, and helping with the transition to climate neutrality and a circular economy. Several catalyst preparation and heterogeneous catalytic processes have limitations and the new high-throughput reactor will help to solve them. Traditional methods for synthesis are neither atom-efficient nor sustainable [2] and most of the studies are developed at laboratory scale. The processing routes for the biomass valorization do not meet still the requirements for industrial needs. The development of new production technologies in an energy and environmentally friendly way is still missing. ${ }^{5}$ The breakthrough processes opened by the high-throughput reactor will allow to study new tools of activation hitherto unexplored in catalysis, which could facilitate the discovery of new chemical reactions and/or new regio-, chemo- and stereo-selectivity patterns, challenging the current paradigms of conventional organic chemistry and catalysis. Among all target products selected, mechanical energy-assisted synthesis has never been used for their preparation to the best of our knowledge.

In this challenging scenario, the biodiesel production needs an effort to develop new technologies to reduce its cost and improve energy efficiency, as well as promote the use of second generation feedstock, not competing with food supply [6,7]. The main challenge must be focused in the change of homogenous to heterogeneous process. The use of a solid catalyst will allow a better separation, reduce the post-treatment needs, reduce the use of water in washing steps, minimize the formation of soaps and produce better quality of both biodiesel and glycerol $[8,9]$. The second difficulty lies in the inmiscibility of vegetable oil and alcohol, which generates mass transfer limitations decreasing the reaction rate. A new method that allows a better mixing of the reactants with scalable opportunity is still missing. In this sense, the new high-throughput reactor seems a good opportunity firstly, for the synthesis of a solid catalyst and secondly, for the heterogeneous transesterification of triglycerides with methanol to produce biodiesel.

\section{Materials and Methods}

\subsection{Preapration of heterogeneous catalyst: calcium diglyceroxide}

$\mathrm{CaO}$ and glycerol were mixed in a batch reactor, before pumping to the mechanochemical reactor. This equipment possesses a chamber containing yttrium-doped zirconia beads and micro-beads that occupies 55-70\% of total volume, and it works with a continuous input flow (between 4 and $150 \mathrm{~L} / \mathrm{h}$ ). Experimental variables, such as methanol:vegetable oil molar ratio, reaction temperature and time, were optimised. The solid catalyst prepared was characterised by several techniques, such as X-ray diffraction, scanning electron microscopy and thermal analysis.

\subsection{Heterogeneoous transesterification reaction with methanol}

The calcium diglyceroxide optimized was tested in the transesterification reaction of vegetable oil with methanol. Kinetics of Fatty Acid Methyl-Esthers (FAME) yield is analyzed by HPLC, comparing conventional batch reactor, based on the use of a 3-neck reactor at laboratory scale (60 $\mathrm{ml}$ of total volume), and high-throughput reactor (H-TR) at $50{ }^{\circ} \mathrm{C}$, a methanol:oil molar ratio of $4: 1$ and $1.5 \%$ of weight percentage of catalyst by oil weight.

\section{Results}

Calcium diglyceroxide is a suitable candidate for the transesterification of triglycerides in heterogeneous conditions; even more, some studies showed that $\mathrm{CaO}$ needs to be transformed to $\mathrm{CaDG}$ during the reaction to be active $[10,11]$. Some synthesis protocols have been published, but in batch reactor and with some hours of residence time [12], so an industrial approach has to be developed and the high-throughput reactor is a good candidate to obtain this objective. 
Several reaction conditions were tested in order to optimize the CaDG production, such as $\mathrm{CaO} /$ glycerol molar ratio, stirring time and temperature (Table 1). Even more, the effect of water presence (10 wt.\%) in glycerol was also evaluated. The percentage of crystalline CaDG formed was quantified by using the Rietveld method. A XRD study was done for all samples and the nominal composition from the structure obtained in the peak identification step was carried out by a Rietveld study. The best fit and convergence of the refinement was obtained taking into account sample displacement, background, peak profile parameters and the scaling of each phase. The percentage of CaDG is shown in Figure 1.

Table 1. Experimental conditions used in the optimisation of the synthesis of CaDG

\begin{tabular}{ccrrcc}
\hline Entry & $\begin{array}{c}\text { Glycerol } \\
\text { Quality } \\
\left.\text { (wt. } \% \mathbf{H}_{\mathbf{2}} \mathbf{O}\right)\end{array}$ & $\begin{array}{c}\text { Stirring } \\
\text { time } \\
\text { (min) }\end{array}$ & $\begin{array}{c}\text { Glyc/CaO } \\
\mathbf{M R}^{*}\end{array}$ & $\begin{array}{c}\text { Temperature } \\
\left.\mathbf{(}^{\circ} \mathbf{C}\right)\end{array}$ & $\begin{array}{c}\text { \% CaDG } \\
\text { (XRD) }\end{array}$ \\
\hline 1 & 0 & 5 & 30 & 25 & 15.40 \\
\hline 2 & 0 & 5 & 30 & 50 & 18.30 \\
\hline 3 & 0 & 5 & 50 & 25 & 18.10 \\
\hline 4 & 0 & 5 & 50 & 50 & 84.30 \\
\hline 5 & 0 & 30 & 30 & 25 & 91.20 \\
\hline 6 & 0 & 30 & 30 & 50 & 99.30 \\
\hline 7 & 0 & 30 & 50 & 25 & 99.50 \\
\hline 8 & 0 & 30 & 50 & 50 & 55.50 \\
\hline 9 & 10 & 5 & 30 & 25 & 82.60 \\
\hline 10 & 10 & 30 & 50 & 50 & 100 \\
\hline
\end{tabular}

${ }^{*}$ The glycerol/CaO molar ratio is multiplied by 10 .

The synthesis was always done with a bead sizes of $0.5 \mathrm{~mm}, 50 \mathrm{wt} \%$ of methanol, as solvent, and without water. We can observe that $30 \mathrm{~min}$ of stirring time, after the high-throughput reactor, is needed to achieve nearly $100 \%$ of $\mathrm{CaDG}$ (entries 6 and 7). Then, if a glycerol/CaO molar ratio of 3 is used, the temperature must be increased up to $50^{\circ} \mathrm{C}$. At room temperature, an excess of glycerol is mandatory (entry 7 ). Moreover, the influence of the presence of water was tested (10 wt\% water in the glycerol phase was added), and it was observed that a similar purity of CaDG is attained (entry 10). Finally, taking into account this preliminary results and with the objective of decreasing the percentage of methanol used to reduce the viscosity of the mixture, the size of beads was shifted to $1 \mathrm{~mm}$, and it is demonstrated that no needs of methanol was necessary to maintain a similar high yield.

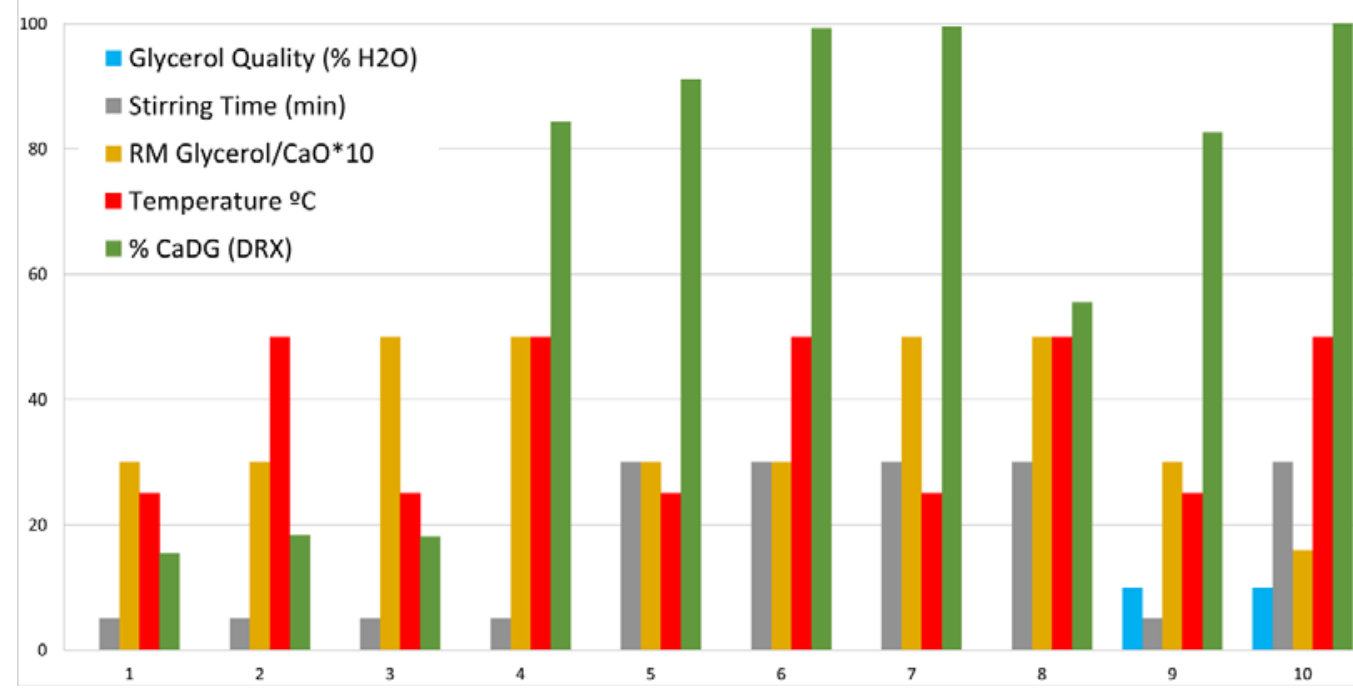

Figure 1. Experimental conditions and percentage of $\mathrm{CaDG}$, as calculated by Rietveld method. 
Therefore, for the first time, a mechanochemical synthesis protocol under flow conditions is described to obtain CaDG in a scalable process to meet industrial needs. The high-throughput reactor, using a flow between 4 and $150 \mathrm{~L} / \mathrm{h}$, allows the synthesis without need of solvent, at low temperature, even in the presence of water [13].

\subsection{Heterogeneoous transesterification reaction with methanol}

In order to overcome the main drawback in biodiesel production, associated to the immiscibility of vegetable oils and methanol which decreases the reaction rate, .alternative processes to the conventional batch system using a homogeneous catalyst, have been previously reported, for instance, by using enzymatic catalysis, supercritical conditions, ultrasound reactors or microwaves [14,15]. But most of these technologies has still limitations to be up-scaled. Taking into account these previous assumption, a methanol:oil molar ratio of 4:1 was used, as well as a low reaction temperature $\left(50{ }^{\circ} \mathrm{C}\right)$ and a catalyst loading of $1.5 \mathrm{wt} . \%$. Figure 2 displays the kinetics of the reaction with both systems. The best performance is reached by using the high-throughput reactor, since, after $1 \mathrm{~h}$ of reaction time, the FAME yield increases from 20 until $60 \mathrm{wt} . \%$ after passing the reaction mixture by the new reactor.

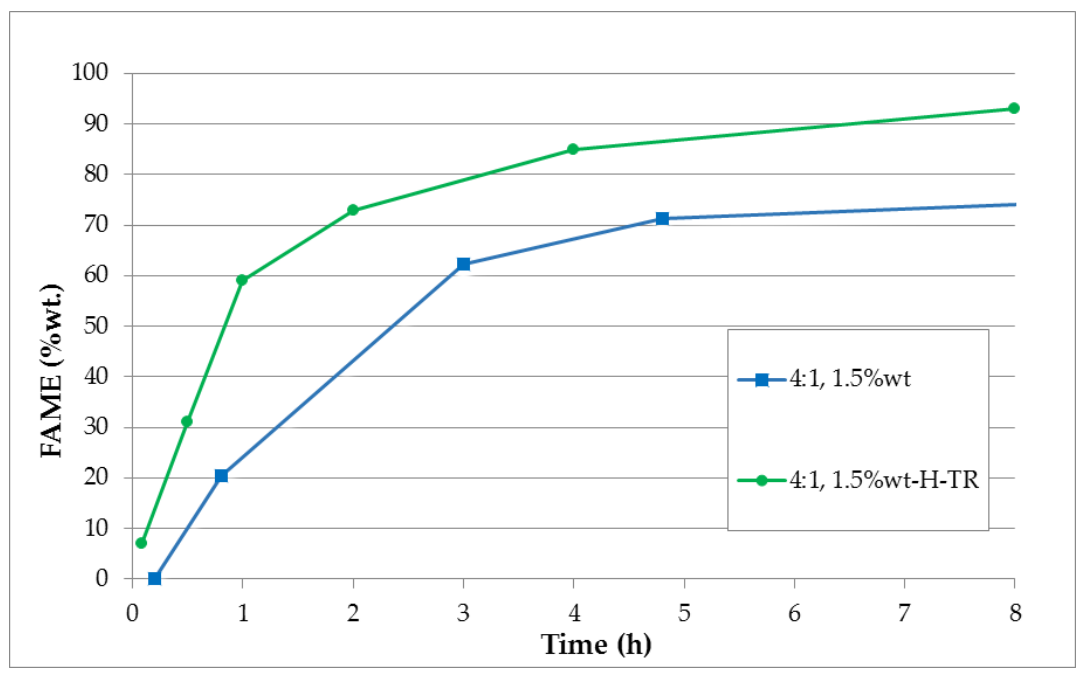

Figure 2. Kinetics of FAME yield under conventional and high-throughput reactor (H-TR) at $50{ }^{\circ} \mathrm{C}, 4 / 1$ methanol:oil molar ratio and $1.5 \%$ of weight percentage of catalyst by oil weight.

Most of the continuous biodiesel production methods, reported so far in the literature, require the use of high reaction temperature and pressure, working with very low flow rates [16], so the use of the high-throughput reactor is very promising, since both low temperature and methanol:oil molar ratio have been used [17].

\section{Conclusions}

In particular, this breakthrough technology will open new opportunities for greener and more efficient chemical processes as it is demonstrated, in biodiesel production and catalyst preparation to: i) shorten reaction times (from $\mathrm{h}$ to $\mathrm{min}$ ) as a result of the activation by mechanical energy; ii) decrease the reaction temperature, since mechanical activation takes place under non-equilibrium conditions; iii) improve reactions kinetics as mass transfer limitations are reduced, while the collision between beads and reactants increases the probability of contact between them; iv) minimize the use of reactants (methanol); v) increase volume treated (from $\mathrm{mL}$ to $\mathrm{L}$ ), enabling a real scale-up; and vi) enhance the yields and/or selectivity, as regeneration of catalyst surface takes place simultaneously to the reaction.

In this sense, the high-throughput reactor allows to optimize the synthesis protocol of calcium diglyceroxide in flow conditions with no use of solvent and no needs of temperature. In addition, the results for the vegetable oil transesterification process are promising, since at a lower temperature they show better biodiesel performance than the conventional batch reactor. 


\section{Patents}

Patent new biodiesel process - WO2018002559: Method for producing fatty acid esters and glycerol at low temperature.

Patent CaDG synthesis - PCT/FR 2017052675 : Procédé de fabrication de diglyceroxide de calcium.

Author Contributions: Irene Malpartida: Writing - Original Draft, Investigation, Data Curation. Pedro Maireles-Torres: Writing - Original Draft, Conceptualization, Project administration. Samy Halloumi : Investigation, Data Curation. Valentin Lair : Investigation, Data Curation. Julien Thiel : Project administration, Resources, Supervision. François Lacoste : Funding acquisition.

Acknowledgments: We thank the technical support of Central Research Services of the University of Málaga.

Conflicts of Interest: The authors declare no conflict of interest, as well as the funders had no role in the design of the study; in the collection, analyses, or interpretation of data; in the writing of the manuscript, or in the decision to publish the results.

\section{References}

1 Rodríguez-Padrón, D.; Puente-Santiago, A.; Balu, A.; Muñoz-Batista, M.; Luque, R.; Environmental catalysis: present and future. Chem Cat Chem, 11, 2019, 18-38

2 Kurosawa, W.; Nakano, T.; Amino Y.; Practical large-scale production of dihydrocapsiate, a nonpungent capsaicinoid-like substance. Biosci, Biotech, and Bioch, 81, 2017, 211.

3 Muñoz-Batista, M. J.; Rodríguez-Padrón, D.; Puente-Santiago, A.; Luque, R.; Mechanochemistry: toward sustainable design of advanced nanomaterials for electrochemical energy storage and catalytic applications, ACS Sustainable Chem. Eng., 6, 8, 2018, 9530-9544.

4 The European Green Deal, Brussels, 11.12.2019 COM(2019) 640 final., 2019

5 Schöppe, H.; Kleine-Möllhoff, P.; Epple, R.; Energy and material flows and carbon footprint assessment concerning the production of HMF and furfural from a cellulosic biomass, Processes, 8, 2020, 119.

6 M.M. Gui, K.T. Lee, S. Bhatia, Feasibility of edible oil vs. non-edible oil vs. waste edible oil as biodiesel feedstock, Energy 33, 2008; 1646-1653

7 I.B. Bankovic-Ilic, O.S. Stamenkovic, V.B. Veljkovic, Biodiesel production from non-edible plant oils, Renew. Sustain. Energy Rev. 16, 2012; 3621-3647

8 A.F. Lee, K. Wilson, Recent developments in heterogeneous catalysis for the sustainable production of biodiesel, Catal. Today 242, 2015; 3-18

9 M. Kouzu, A. Fujimori, R. ta Fukakusa, N. Satomi, S. Yahagi, Continuous production of biodiesel by the CaO-catalyzed transesterification operated with continuously stirred tank reactor, Fuel Process. Technol. 181, 2018; 311-317

10 M. Kouzu, T. Kasuno, M. Tajika, S. Yamanaka, J. Hidaka, Active phase of calcium oxide used as solid base catalyst for transesterification of soybean oil with refluxing methanol, Appl. Catal. Gen. 334, 2008; 357-365

11 A. Buasri, M. Lukkanasiri, R. Nernrimnong, S. Tonseeya, K. Rochanakit, W. Wongvitvichot, U. Masa-ard, V. Loryuenyong, Rapid transesterification of Jatropha curcas oil to biodiesel using novel catalyst with a microwave heating system, Kor. J. Chem. Eng. 33, 2016; 3388-3400

12 I. Lukic, Z. Kesic, M. Zdujic, D. Skala, Calcium diglyceroxide synthesized by mechanochemical treatment, its characterization and application as catalyst for fatty acid methyl esters production, Fuel 165, 2016; 159-165

13 Patent CaDG synthesis - PCT/FR 2017052675 : Procédé de fabrication de diglyceroxide de calcium.

14 M. Kouzu, J.S. Hidaka, Transesterification of vegetable oil into biodiesel catalyzed by CaO: a review, Fuel 93, 2012; 1-12 
15 I. Reyero, G. Arzamendi, L.M. Gandía, Heterogenization of the biodiesel synthesis catalysis: CaO and novel calcium compounds as transesterification catalysts, Chem. Eng. Res. Des. 92, 2014; 1519-1530

16 Y. Xiao, L. Gao, G. Xiao, B. Fu, L. Niu, Experimental and modeling study of continuous catalytic transesterification to biodiesel in a bench-scale fixed-bed reactor, Ind. Eng. Chem. Res. 51, 2012; 11860-11865.

17 Patent new biodiesel process - WO2018002559: Method for producing fatty acid esters and glycerol at low temperature.

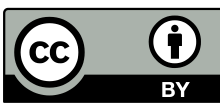

C 2019 by the authors; licensee MDPI, Basel, Switzerland. This article is an open access article distributed under the terms and conditions of the Creative Commons Attribution (CC-BY) license (http://creativecommons.org/licenses/by/4.0/). 